\title{
Assessing the validity and reliability of the Turkish version of the Trunk Impairment Scale in stroke patients
}

\author{
(1) Sinem Sag, ${ }^{1}$ () Raikan Buyukavci, ${ }^{2}$ (I) Fusun Sahin, ${ }^{3}$ \\ (D) Mustafa Serdar Sag, ${ }^{1}$ (D) Beril Dogu, ${ }^{4}$ (D) Banu Kuran 4 \\ ${ }^{1}$ Division of Rheumatology, Department of Physical Medicine and Rehabilitation, Sakarya University Faculty of Medicine, Sakarya, Turkey \\ 2Department of Physical Medicine and Rehabilitation, Inonu University Faculty of Medicine, Malatya, Turkey \\ ${ }^{3}$ Department of Physical Medicine and Rehabilitation, Pamukkale University Faculty of Medicine, Denizli, Turkey \\ ${ }^{4}$ Department of Physical Medicine and Rehabilitation, Sisli Hamidiye Etfal Training and Research Hospital, Istanbul, Turkey
}

\begin{abstract}
OBJECTIVE: To determine the validity and reliability of the Turkish version of the Trunk impairment scale (TIS), used in the evaluation of somatic, motor, and coordination disturbances in stroke patients, and provide a culturally adapted version for use in the Turkish population.

METHODS: A total of 80 patients who were either hospitalized at our facility and rehabilitated for stroke or admitted at our outpatient clinics were included in this study. Reliability was evaluated by the internal consistency (Cronbach a) and test reproducibility [intra-class correlation coefficient (ICCC)] methods, and validity was evaluated by the correlation between subgroups and the total scores of the TIS and Berg Balance Scale (BBS), Brunnstrom phases, Barthel index (BI), Rivermead mobility index (RMI), and Short Form-36 (SF-36) scores.

RESULTS: The mean age of the patients was $63.00 \pm 12.1$ years. Out of a total of 80 subjects, 34 were female and 46 were male. The reliability of the scale was evaluated by the internal consistency, inter- and intra-observer reliability, and test reproducibility. The findings showed that the Turkish form of the scale was reliable at a good level. The test values were as follows; Cronbach a: >0.70, ICCC: $0.969-1$, subgroups and total score comparison: 0 . The correlation between TIS and BBS was considerably high in the validity analysis $(p<0.001)$. Further, significant associations among the BI, RMI, KF-36, Brunnstrom, and TIS scores were found $(p<0.001)$, which indicate the structural validity of this scale.

CONCLUSION: TIS is a scale used in measuring the motor derangement that develops after a stroke. It has sufficient reliability, internal consistency, and validity for use in clinical practice and stroke investigations. Our study has shown that TIS used for the evaluation of body balance is valid and reliable for the Turkish population.
\end{abstract}

Keywords: Reliability and validity; stroke; trunk impairment scale.

Cite this article as: Sag S, Buyukavci R, Sahin F, Sag MS, Dogu B, Kuran B. Assessing the validity and reliability of the Turkish version of the Trunk Impairment Scale in stroke patients. North Clin Istanb 2019;6(2):156-165.

troke is a significant health issue worldwide. According to European and American statistics, it is the third most frequent cause of death after heart disease and cancer. Every year, millions of individuals are disabled globally due to this disorder. Stroke patients face a plethora of medical, economic, and social problems every day.

Changes in posture and balance are frequently seen in hemiparesis caused by stroke, with derangements

Received: November 09, 2017 Accepted: March 07, 2018 Online: August 14, 2018

Correspondence: Dr. Sinem SAG. Sakarya Universitesi Tip Fakultesi, Fiziksel Tedavi ve Rehabilitasyon Anabilim Dali, Romatoloji Bilim Dali, Sakarya, Turkey.

Tel: +905065360355 e-mail: drsinemyamac@yahoo.com

(c) Copyright 2019 by Istanbul Provincial Directorate of Health - Available online at www.northclinist.com

(c) (1) (8) 
observed while sitting and standing both. Many studies have shown that the static sitting balance is an early predictor of functioning in stroke patients $[1,2]$. Various questionnaires have been used to measure trunk performance and balance in stroke patients. These scales should be adapted to the language and culture of countries where different languages are spoken [3].

The trunk impairment scale (TIS) was developed by Geert Verheyden in 2003 for the evaluation of motor derangements that occur after a stroke. It aims to measure the quality of trunk movements and provide guidance for treatment. It is also used in the evaluation of trunk balance in disorders, such as Parkinson's disease, multiple sclerosis, and cerebral palsy, in addition to stroke patients, which proves its validity and reliability [4-6].

The aim of this study, therefore, was to assess the validity and reliability of the Turkish version of the TIS that is used in the evaluation of somatic motor and coordination disturbances in stroke patients and also provide a culturally adapted version of the scale for use in the Turkish population.

\section{MATERIALS AND METHODS}

This study was approved by the Ethics Committee of Sisli Etfal Hospital for Training and Research (Approval number: 71). Written informed consents were obtained from all patients enrolled in the study.

\section{Patient Selection}

A total of 80 patients who were hospitalized for stroke rehabilitation in our facility or admitted at our outpatient clinics were included in this study. The exclusion criteria were as follows: presence of major sensory or cognitive derangement (Mini-mental state examination score $<16$ ), absence of the ability to understand instructions, presence of serious vision problems, presence of orthopedic problems that may preclude exercises that the patient is required to perform lying down, and a mother tongue other than Turkish.

\section{Evaluation Measures}

The information we collected included the demographic data of enrolled patients such as; age, gender, educational status and occupation; and the clinical data, such as duration of illness, type of lesion, location of the lesion, involved side, and presence of co-morbidities.
Cognitive Functions: Cognitive functions of the patients were evaluated with the Mini-Mental State Examination (MMSE) that was first developed by Folstein in 1975 [7]. It has since then been revised and the validity of its Turkish version has been shown before [8]. Patients with an MMSE score $<16$ were excluded from the study.

Activities of Daily Living: The daily living activity levels and physical independence of the patients were evaluated by the Barthel Index (BI), which has proven its validity and reliability in various patient groups and populations. This scale mainly evaluates the state of mobility and ability to perform self-care activities. It includes 10 sections: nourishment, transfer, self-care, use of WC, bathing, movement, use of a wheelchair (if relevant), climbing up/down the stairs, putting on clothes, and bowel/bladder control. The final score can be between 0 and 100. Its validity and reliability in our society have been shown before [9].

Evaluation of General Quality of Life: Short form-36 (SF-36) was used to evaluate the quality of life. It was developed by Ware in 1987.SF-36 is a form that consists of eight subscales (physical functioning, physical role limitation, emotional role limitation, social functioning, pain, vitality, mental health and general health), each containing 36 items aiming to determine health status in clinical investigations, monitor the outcomes of medical care, and evaluate the quality of life [10]. SF-36 uses a total score of 100 points, and each subscale can be scored between 0 and 10. Higher scores in this scale reflect a better level of health, whereas lower scores show deterioration in health. The validity and reliability of the Turkish version have been shown before [11].

Evaluation of Motor Functions: Neurophysiologic evaluation was done according to the Brunnstrom improvement stages. The Brunnstrom Approach is used to assess motor improvement in the upper extremity, hand, and lower extremity, and at the same time, to determine the current motor stage of stroke patients. The Brunnstrom approach is a scale of six stages, which includes movement patterns where improvement progressively increases for each region. Higher scores reflect better motor improvement [12].

Evaluation of Mobility: The Rivermead Mobility Index (RMI) was used to measure the mobility of patients. This is an index with one dimension that is focused on measuring the mobility status and includes activities of basic mobility [13]. It includes 14 questions from the Guttman Scale, 1 observation and a hierarchic series of 
activities from "turning in bed" to "running" Total $15 \mathrm{ac}$ tivities are used in the evaluation, and each "yes" answer yields one point. A total score of 15 points indicates an absence of mobility problems, whereas $<14$ means that there is a mobility problem. As the RMI is a hierarchic structure (from simple to complex), a smaller score indicates a more severe problem [14].

Evaluation of Balance: The Berg Balance Scale (BBS) was used to evaluate the balance of stroke patients. The test evaluates the ability of patients to maintain their balance during functional activities. It consists of 14 items, and each item is scored between 0 (bad) and 4 (best) points. It assesses dependence and/or independence while sitting, standing, standing with feet together, standing at the tandem position, balancing on one foot, and the ability of an individual to change positions. The highest score at the BBS reflects the proper balance. Cases are classified into groups having a "high risk of falling" (score: 0-20 points), "medium risk of falling" (21-40 points), or "low risk of falling" (41-56 points) according to the scores of this test $[15,16]$. The Turkish version of the BBS has been shown to be reliable, valid and sensitive to change in Turkey [17].

Evaluation of Motor Disturbances: The TIS was developed by Geert Verheyden in 2003 for the evaluation of motor derangements that occur after a stroke. It aims to measure the quality of trunk movements and provide guidance for treatment. It is also used in the evaluation of trunk balance in disorders, such as Parkinson's disease, multiple sclerosis, and cerebral palsy, in addition to stroke patients, which proves its validity and reliability $[3,4,6]$. We have endeavored to translate the TIS, which is used in the evaluation of motor and coordination disturbances of the trunk in stroke patients, determines the validity and reliability of the Turkish version and provides its cultural adaptation for use in the Turkish population.

\section{TIS Translation and Adaptation}

The suggestions of Guillemin et al., Beaton et al. and the EORTC Quality of Life Group was used in the creation of the Turkish version of the scale, before translation and cultural adaptation [18-20].

In the first step, two individuals whose mother tongue was Turkish and who were fluent in English independently translated the TIS from English to Turkish. The differences between the two Turkish translations were evaluated and corrected by a physical therapy specialist whose mother tongue was Turkish and who knew Eng- lish at an advanced level, and a common Turkish form was created. In the second step, this Turkish form was translated into English again and re-translated to Turkish by two persons who knew English at an advanced level. The differences were corrected, and a satisfactory harmony with the original scale was obtained.

At the cultural adaptation level, the translations by two individuals with good knowledge of English were evaluated by an experienced physiotherapist. The differences were corrected, and the resulting Turkish version was evaluated by eight physiotherapists, who were unable to detect any mistakes or unclear sentences. This last version of the Turkish translation was picked as the final scale.

\section{Validity and Reliability Examination}

The Turkish version of the TIS was applied to 80 participants twice in one week to test its validity and reliability. Reliability is defined as the precision and reproducibility of a scale [21]. To determine the inter-observer reliability, 20 individuals were selected out of the 80 participants and were examined by two different observers at an interval of approximately 15-30 minutes. For intraobserver reliability, these 20 individuals were evaluated by the same observer twice a day: in the morning and in the evening.

Validity is defined as the degree with which a scale can measure its aim [21]. The validity of the TIS was evaluated by ascertaining its structural validity. To evaluate structural validity, correlations between the subsections and the total scores of TIS and Brunnstrom stages, along with the BI, BBS, RMI, and SF-36 scores were evaluated.

\section{Statistical Evaluation}

The data obtained in this study was transferred to the SPSS ver. 13.0 software and statistical analysis was done using this program. Descriptive methods (mean, standard deviation) were used in the evaluation of the demographic characteristics of the participants. The internal consistency of the scale was evaluated with the Cronbach alpha $(\alpha)$ coefficient, and the intra- and inter-observer reliability was evaluated with the Cronbach $\alpha$ coefficient and intra-class correlation coefficient (ICCC). The testrepeat test reliability of the scale was evaluated with the Cronbach $\alpha$ coefficient, intra-class correlation coefficient (ICCC), and Spearman correlation.

The criteria validity of the scale was evaluated with the Pearson correlation according to the BBS. Its structural 
TABLE 1. Demographic characteristics of patients $\mathrm{n}$

\section{Gender \\ Female \\ Male \\ Total \\ Age}

Time since stroke (months)

Occupation

House wife

Civil servant

Worker

Freelance

Total

Educational status

Left primary school

Primary school

Junior high school

High school

University

Total

Lesion location

MCA

PCA

ACA

LCA

Pons

Thalamus

Other

Lesion type

Thromboembolic

Haemorrhagic

Total

Involved side

Right

Left

Total

Co-morbidity

None

Hypertension (HT)

Diabetes Mellitus (DM)

Cardiac disease

Other

ACA: Anterior cerebral artery; MCA: Middle cerebral artery PCA: Potsrior cerebral

artery; LCA: Lentrıculostriat artery.

11.2

100.0

47.5

52.5

100.0

15.0

60.0

30.0

22.5

16.3 validity was evaluated with BI, RMI, SF-36, Brunnstrom stages, and Pearson correlation. The correlation coefficient $(r)$ was considered very weak if it fell between 0.00 and 0.25 ; weak if it fell between 0.26 and 0.49 , medium if it fell between 0.50 and 0.69 , high if it fell between 0.70 and 0.89 , and very high if it fell between 0.90 and 1.00. The results were evaluated according to the $\mathrm{p}<0.05$ significance level at $95 \%$ confidence intervals.

\section{RESULT}

Total 80 patients (34 females and 46 males) who were interned for hemiplegia rehabilitation were included in our study. The age, gender, occupation, educational status, additional diseases and other demographic characteristics of the patients are summarized in Table 1.

Of the 80 patients included in the study, $73 \%$ had cerebrovascular thromboembolism, $7 \%$ had cerebrovascular bleeding, 26\% had MCA, $13 \%$ had thalamus, $11 \%$ had LCA, $8 \%$ had PCA, and 3\% had ACA infarction. In addition, 38 patients had right hemiplegia, 42 had left hemiplegia, $60 \%$ had hypertension as an additional disease, $30 \%$ had diabetes mellitus, and $22 \%$ had ischemic heart disease.

The results of the patients' Brunnstrom staging, MMSE, BI, RMI, and BBS are summarized in Table 2.

The Cronbach $\alpha$ values used to evaluate the internal consistency were over 0.70 for all parameters and total scores of TIS (Table 3). This value was 0.77 for the static sitting balance, 0.90 for the dynamic sitting balance, 0.85 for coordination, and 0.93 for the TIS total score. These values show that the TIS has a high internal consistency.

The ICCC for subgroups used in the test-repeat test reliability was between 0.907 and 0.995 , and the TIS to-
TABLE2. Mean scores of patients' Mini-Mental test, Brunnstrom staging, Rivermead, Barthel and Berg Balance tests

Mean \pm SD

Mini mental test

$20.19 \pm 3.772$

Brunnstrom upper extremity

$3.60 \pm 1.514$

Brunnstrom hand

$3.35 \pm 1.677$

Rivermead index

$6.5 \pm 3.82$

Barthel index

$60.81 \pm 24.88$

Berg balance scale

$33.93 \pm 19.73$
SD: Standard deviation. 
TABLE 3. Trunk Instability Scale showing Internal Consistency

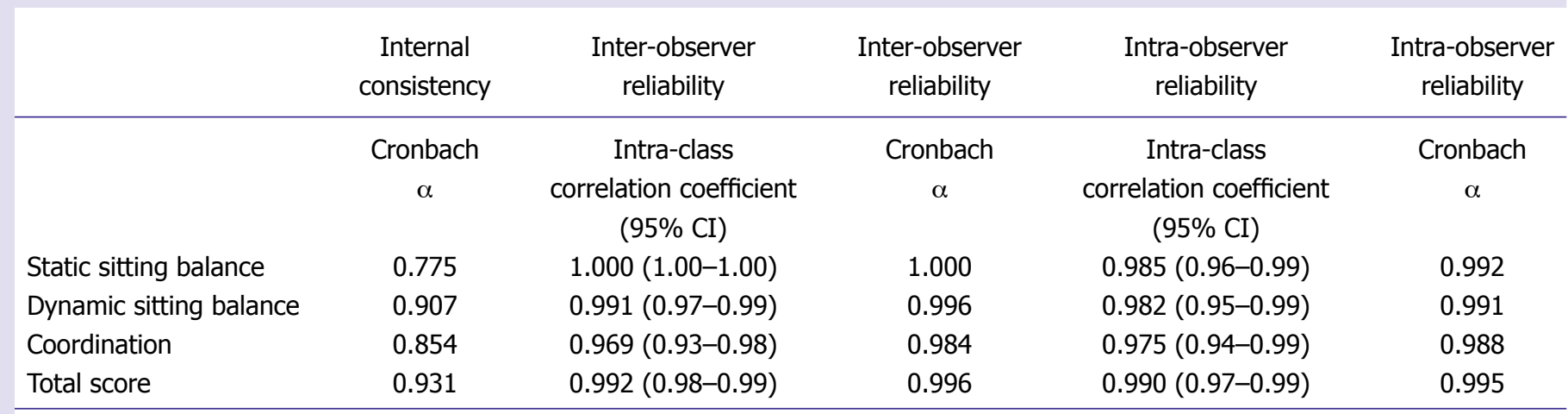

CI: Confidence interval.

TABLE 4. Trunk Impairment Scale showing Test-repeat Test Reliability

Intra-class correlation

coefficient (ICCC)
Spearman correlation*

0.995 (0.99-1.00)

$1.000(1.0-1.0)$

$0.980(0.96-0.98)$

$0.991(0.98-0.99)$

Static sitting balance 3

Dynamic sitting balance total

Dynamic sitting balance 1

Dynamic sitting balance 2

Dynamic sitting balance 3

Dynamic sitting balance 4

Dynamic sitting balance 5

Dynamic sitting balance 6

Dynamic sitting balance 7

Dynamic sitting balance 8

Dynamic sitting balance 9

Dynamic sitting balance 10

Coordination total

Coordination 1

Coordination 2

Coordination 3

Coordination 4

TIS total score
0.907 (0.86-0.94)

$0.563(0.39-0.70)$

$0.636(0.49-0.75)$

$0.687(0.55-0.78)$

$1.000(1.0-1.0)$

$0.757(0.64-0.83)$

$0.849(0.77-0.90)$

$0.890(0.83-0.92)$

$1.000(1.0-1.0)$

$0.687(0.55-0.79)$

$0.904(0.85-0.93)$

0.961 (0.94-0.97)

$0.874(0.81-0.92)$

$0.951(0.93-0.97)$

$0.960(0.94-0.97)$

$0.924(0.88-0.95)$

0.964 (0.94-0.98)
Internal consistency

(Cronbach alpha)

ICCC: Intra-class correlation coefficient; TIS: Trunk impairment scale; ${ }^{*} \mathrm{p}<0.001$.

tal score was 0.964 (Table 4). The intra-observer reliability, which we calculated with ICCC, was 0.98 for static sitting balance, 0.98 for dynamic sitting balance, 0.97 for coordination, and 0.99 for total TIS score. The inter-observer reliability coefficient was 1.0 for the static sitting balance, 0.99 for the dynamic sitting balance, 0.98 for 
TABLE 5. Correlation among TIS, BBS, RMI, and BI scales

\begin{tabular}{|c|c|c|c|}
\hline TIS & BBS & RMI & BI \\
\hline \multicolumn{4}{|c|}{ Static sitting balance } \\
\hline r value & 0.560 & 0.507 & 0.420 \\
\hline$p$ value & $<0.0001$ & $<0.0001$ & $<0.00011$ \\
\hline \multicolumn{4}{|c|}{ Dynamic sitting balance } \\
\hline r value & 0.901 & 0.784 & 0.812 \\
\hline$p$ value & $<0.0001$ & $<0.0001$ & $<0.0001$ \\
\hline \multicolumn{4}{|c|}{ Coordination } \\
\hline r value & 0.806 & 0.713 & 0.730 \\
\hline$p$ value & $<0.0001$ & $<0.0001$ & $<0.0001$ \\
\hline \multicolumn{4}{|l|}{ Total score } \\
\hline r value & 0.887 & 0.783 & 0.780 \\
\hline$p$ value & $<0.0001$ & $<0.0001$ & $<0.0001$ \\
\hline
\end{tabular}

r: Pearson correlation coefficient; TIS: Trunk impairment scale; BBS: Berg balance scale RMI: Rivermead mobility index BI: Barthel index.

coordination, and 0.99 for the total TIS score. These results show that inter-observer consistency is significant.

A strong positive association was found between the TIS and the BBS, and the BI and RMI sub-scores and total score $(p<0.001$; Table 5$)$. The relationship between the BBS and static sitting balance was positive and significant at a medium level of significance $(r=0.56)$, and was similarly positive with the dynamic sitting balance $(\mathrm{r}=0.90)$, coordination $(\mathrm{r}=0.80)$, and total TIS score $(r=0.88)$. When we looked at the TIS and RMI correlation, we found a positive association of medium significance between the static sitting balance and RMI $(\mathrm{r}=0.50)$ and a highly significant association between the dynamic sitting balance $(\mathrm{r}=0.78)$, coordination $(\mathrm{r}=0.71)$ and the TIS total score $(r=0.78)$ with RMI $(\mathrm{p}<0.001)$.

An association was not found between the physi$\mathrm{cal}$ and emotional role limitations in SF-36 subsections and TIS sub-scores and total scores $(\mathrm{p}>0.05)$, whereas a significant association was shown between other SF36 subsections and TIS total scores $(\mathrm{p}<0.05$; Table 6$)$. A parallel increase in the TIS total score was detected, increasing the Brunnstrom upper extremity, hand, and lower extremity stage values $(\mathrm{p}<0.01)$.

\section{DISCUSSION}

This study has shown that the Turkish version of the TIS, which is used in the assessment of trunk balance in stroke patients, is valid and reliable in the Turkish population.

Stroke is a socio-medical problem which threatens the quality of life, may be fatal, and is most frequently encountered among neurologic disorders as the third cause of death after heart disease and cancer, according to data from developed countries. It is considered the first cause of morbidity, and its importance progressively increases with age. As the disorder that most frequently causes disability, its rehabilitation is essential [22].

Stroke patients generally have difficulty in maintaining balance, experience postural disturbance, are unable to hold the head and trunk at the same plane, and have disrupted weight distribution. Many physical functions require sufficient balance and trunk control is necessary for maintaining body position and remaining stable after the position changes, which define the ability to accomplish daily activities and mobility [23]. The sitting balance was shown to be a marker of motor and functional improvement after stroke [24, 25]. Trunk control was found to be decreased in stroke patients in comparison to age and gender-matched healthy control groups, and $45 \%-70 \%$ of trunk control was shown to be lost in the acute stages of stroke [26].

The ability to control trunk balance in sitting and daily living activities is important for successful rehabilitation [27]. Scales that aim to determine trunk balance do not reflect improvement by themselves. We have to evaluate functions of trunk muscles not only in terms of disability but also in terms of impairment to better understand improvement in trunk balance after stroke and develop more active treatment programs in patients with poor trunk balance.

The TIS, which was developed by Verheyden et al, is a scale that evaluates the functions of trunk muscles at impairment level. In a study aiming to determine the discriminatory ability of the TIS, healthy individuals were compared to stroke patients. A prominent difference was found between the two groups in terms of the total TIS scores and sub-scores. Higher scores were found in healthy individuals as compared to stroke patients. In conclusion, it was found to be a scale with an ability to discriminate between stroke patients and healthy individuals [28]. Total 32 articles were included in an interview investigating clinical scales that measured trunk performance after stroke. In this comparative study evaluating the psychometric features of scales, the TIS was found to be a very effective scale for measurement in the evaluation of trunk performance after stroke [3]. 
TABLE 6. Correlation between TIS scale and SF-36 scale

TIS

\begin{tabular}{|c|c|c|c|c|}
\hline \multirow{2}{*}{ SF-36 } & \\
\hline & Static sitting balance & Dynamic sitting balance & Coordination & Total score \\
\hline \multicolumn{5}{|c|}{ Physical functioning } \\
\hline r value & 0.250 & 0.565 & 0.558 & 0.595 \\
\hline $\mathrm{p}$ value & $0.026^{*}$ & $0.0001^{* *}$ & $0.0001 * *$ & $0.0001^{* *}$ \\
\hline \multicolumn{5}{|c|}{ Physical role limitation } \\
\hline r value & 0.116 & -0.134 & 0.035 & -0.027 \\
\hline $\mathrm{p}$ value & 0.305 & 0.235 & 0.759 & 0.813 \\
\hline \multicolumn{5}{|l|}{ Pain } \\
\hline r value & 0.277 & 0.325 & 0.278 & 0.331 \\
\hline $\mathrm{p}$ value & $0.013^{*}$ & $0.003 * *$ & $0.012^{*}$ & $0.003 * *$ \\
\hline \multicolumn{5}{|c|}{ General Health } \\
\hline rvalue & 0.106 & 0.365 & 0.387 & 0.376 \\
\hline $\mathrm{p}$ value & 0.349 & $0.001^{* *}$ & $0.0001^{* *}$ & $0.001^{* *}$ \\
\hline \multicolumn{5}{|l|}{ Vitality } \\
\hline r value & 0.116 & 0.210 & 0.243 & 0.221 \\
\hline $\mathrm{p}$ value & 0.305 & 0.062 & $0.0001^{* *}$ & $0.049 *$ \\
\hline \multicolumn{5}{|c|}{ Social functioning } \\
\hline r value & 0.287 & 0.307 & 0.341 & 0.352 \\
\hline $\mathrm{p}$ value & $0.010^{*}$ & $0.006^{* *}$ & $0.002 * *$ & $0.001^{* *}$ \\
\hline \multicolumn{5}{|c|}{ Emotional role } \\
\hline r value & 0.116 & -0.134 & 0.035 & -0.027 \\
\hline $\mathrm{p}$ value & 0.305 & 0.235 & 0.759 & 0.813 \\
\hline \multicolumn{5}{|c|}{ Mental health } \\
\hline r value & 0.115 & 0.272 & 0.228 & 0.252 \\
\hline $\mathrm{p}$ value & 0.308 & $0.015^{*}$ & $0.042^{*}$ & $0.024 *$ \\
\hline \multicolumn{5}{|l|}{ Total score } \\
\hline r value & 0.081 & 0.611 & 0.588 & 0.603 \\
\hline$p$ value & $0.001 * *$ & $0.001^{* *}$ & $0.001 * *$ & $0.001^{* *}$ \\
\hline
\end{tabular}

TIS: Trunk impairment scale; r: Spearman's Correlation coefficient ${ }^{*} \mathrm{p}<0.05 ;{ }^{* *} \mathrm{p}<0.001$.

There have been studies in which the TIS was used as an outcome criterion. In a randomized controlled study investigating the effect of exercises on trunk performance after stroke, trunk exercises were advised in addition to conventional treatment in stroke patients. It was found that when the TIS was used as an evaluation measure, a significant improvement was observed in the dynamic sitting balance subscale of the TIS, which selectively measures lateral flexion of the trunk [29].

Validity and reliability analyses are done to examine the accuracy of data obtained by scales that are used in the field of rehabilitation. Analyses methods to show re- liability, such as internal consistency, test-repeat test reliability, and inter-observer reliability, are also used. The items that make up the scale measure the same structures in relation with one other, each item representing the conceptual structure to be measured to show the internal consistency of the scale.

The internal consistency of a scale shows the degree to which the items reflect the concept to be measured and the relationship between the items that make up the scale. This is determined as a Cronbach $\alpha$ value. In this instance, the $\alpha$ value is between 0 and 1 , and its closeness to 1 reflects a stronger internal consistency. A high in- 
ternal consistency supports the reliability of a scale. We investigated the internal consistency of the subscales of the TIS by calculating the Cronbach $\alpha$ values of static, dynamic sitting balance and coordination subscales of the TIS. This value was 0.77 for the static sitting balance, 0.90 for the dynamic sitting balance, 0.85 for coordination, and 0.93 for the TIS total score. These values show that the TIS has a high internal consistency. This value was 0.79 for the static sitting balance, 0.86 for the dynamic sitting balance, 0.65 for coordination, and 0.89 for the TIS total score. Our results are consistent with the original study [30].

As the patients may be followed up by the same or different physicians at different times, the inter-observer and intra-observer reliability of the scales should also be determined. In the study by Verheyden in 2003, where the TIS was first developed, the test was administered by two different observers to 28 stroke patients, and interobserver reliability was assessed. The inter-observer reliability coefficient was 0.99 for the static sitting balance, 0.98 for the dynamic sitting balance, 0.85 for coordination, and 0.99 for the TIS total score [30]. In our study, the inter-observer reliability coefficient was 1.0 for the static sitting balance, 0.99 for the dynamic sitting balance, 0.98 for coordination, and 0.99 for the TIS total score; these are consistent with the original study.

In the study examining the validity and reliability of the TIS in patients with multiple sclerosis, the interobserver reliability coefficient was 0.95 (total TIS) [5]. In the study investigating the validity and reliability of the TIS in patients with Parkinson's disease, the interobserver reliability coefficient was 0.97 (total TIS) [4]. In the study where the validity and reliability of the TIS were examined in patients with traumatic brain damage, the inter-observer reliability coefficient was 0.95 (total TIS) [31].

The intra-observer reliability is one of the methods that test the reliability of the scale. In the validity and reliability studies with the TIS, there were no studies where the intra-observer reliability was examined, including the original study. The intra-observer reliability results of our study are satisfactory. The intra-observer reliability, which we have calculated as ICCC, was 0.98 for the static sitting balance, 0.98 for the dynamic sitting balance, 0.97 for coordination, and 0.99 for the TIS total score.

In our study, SIKK was 0.99 for static sitting balance for the test-repeat test reliability of the TIS, 0.90 for the dynamic sitting balance, 0.96 for coordination and 0.96 for the total TIS score. In the original study, these values were was 0.91 for the static sitting balance, 0.94 for the dynamic sitting balance, 0.87 for coordination, and 0.96 for the TIS total score [30]. Our results are consistent with the original study.

In the validity and reliability studies of the TIS in patients with multiple sclerosis for test-repeat test reliability ICCC for TIS, the total score was calculated as 0.95 [5]. In the validity and reliability studies of the TIS in patients with Parkinson's disease for test-repeat test reliability ICCC for TIS, the total score was 0.95 [4]. In the study of the validity and reliability of the TIS in patients with traumatic brain damage for test-repeat test reliability ICCC for TIS, the total score was 0.88 [31].

The correlation between the TIS and the BBS was investigated with the Pearson correlation. The static sitting balance is a subscale of the TIS which only measures the independent sitting ability of the patient and does not evaluate other movements, such as lateral flexion and rotation of the trunk, which includes three items. For this reason, in the acute phase, even patients with a very limited ability of movement may obtain high scores. Even if they obtain high scores from the static sitting balance subscale, they may not obtain adequate scores from the dynamic sitting balance, coordination scales, and BDO. This may explain the relationship of the medium significance level between the static sitting balance and BDO, in spite of the highly significant relationship between the dynamic sitting balance and Coordination with the BBS.

To show the structural validity of the TIS, we also examined correlations between the RMI, SF-36, BG, Brunnstrom staging and TIS in our study. Trunk balance is one of the main factors for mobility. For this reason, when we looked at the TIS and RMI correlation, we found a positive association of medium significance between the static sitting balance and RMI $(r=0.50)$ and a very significant association between the dynamic sitting balance $(r=0.78)$, coordination $(r=0.71)$ and the TIS total score $(r=0.78)$ with RMI $(p<0.001)$. We can explain these results with our evaluations of BBS-TIS correlations.

Because we feel that the trunk balance of patients may be associated with mobilization, ambulation and, hence, quality of life, we evaluated the correlation between the TIS and SF-36 in our study. Statistically significant positive correlations were found between the TIS total score and six of the eight subgroups of SF-36 (excluding role 
difficulty and emotional role strength). The positive correlation between physical functioning and the TIS total score was stronger than other subgroups $(r=0.595$, $\mathrm{p}=0.0001)$. Statistically significant positive correlations were found between the dynamic sitting balance and coordination among TIS sub-parameters with the other subgroups (excluding role difficulty and emotional role strength). In addition to these, a statistically significant correlation was not found between the dynamic sitting balance and the vitality sub-group of SF-36 ( $r=0.210$, $\mathrm{p}=0.062$ ). When we looked at the static sitting balance, we found statistically significant positive correlations with only physical functioning, pain and social functioning subgroups $(r=0.250, r=0.277, r=0.287$, respectively), without significant positive correlations with other subgroups.

We evaluated the correlation between the Barthel index (BI) and the TIS for the validity of the TIS in our study. We found a very significant correlation between the BI and the TIS ( $r=0.78)$. In Verheyden's study, a very significant correlation was found between the $\mathrm{BI}$ and the TIS ( $r=0.86)$ [30]. Our result is sufficient. In a multicenter study, the TIS in the sixth month after the stroke and the static sitting balance subscale were found to be important predictors of the $\mathrm{BI}[32]$.

The Brunnstrom is a scale evaluating the developmental stages of movements of functionality after patients experience a stroke. As it is a scale that coordinates the muscle strength of the extremities and trunk, there may be an association between the TIS and Brunnstrom scales. For this reason, we evaluated the correlation between the TIS and Brunnstrom scales in our study and found associations of medium significance between the TIS and three Brunnstrom parameters $(\mathrm{p}<0.01)$.

In conclusion, the TIS is a new scale measuring the motor derangements of the trunk after a stroke. It has sufficient reliability, internal consistency, and validity for use in clinical practice and stroke investigations Our study has shown that the Turkish version of the TIS, which is used in the assessment of trunk balance in stroke patients, is valid and reliable in the Turkish population.

Conflict of Interest: The authors declare no conflict of interest.

Financial Disclosure: The authors declared that this study has received no financial support.

Authorship Contributions: Concept - SS; Design - BK; Supervision - BK; Materials - RB; Data collection and/or processing - SS, RB; Analysis and/or interpretation - SS, MSS, BD, FS; Writing - SS; Critical review - BK, BD, FS.

\section{REFERENCES}

1. Wade DT, Skilbeck CE, Hewer RL. Predicting Barthel ADL score at 6 months after an acute stroke. Arch Phys Med Rehabil 1983;64:24-8.

2. Loewen SC, Anderson BA. Predictors of stroke outcome using objective measurement scales. Stroke 1990;21:78-81. [CrossRef]

3. Verheyden G, Nieuwboer A, Van de Winckel A, De Weerdt W. Clinical tools to measure trunk performance after stroke: a systematic review of the literature. Clin Rehabil 2007;21:387-94. [CrossRef]

4. Verheyden G, Willems AM, Ooms L, Nieuwboer A. Validity of the trunk impairment scale as a measure of trunk performance in people with Parkinson's disease. Arch Phys Med Rehabil 2007;88:1304-8.

5. Verheyden G, Nuyens G, Nieuwboer A, Van Asch P, Ketelaer P, De Weerdt W. Reliability and validity of trunk assessment for people with multiple sclerosis. Phys Ther 2006;86:66-76. [CrossRef]

6. Pham HP, Eidem A, Hansen G, Nyquist A, Vik T, Sxther R. Validity and Responsiveness of the Trunk Impairment Scale and Trunk ControlMeasurement Scale in Young Individuals with Cerebral Palsy. Phys Occup Ther Pediatr 2016;36:440-52. [CrossRef]

7. Folstein MF, Folstein SE, McHugh PR. "Mini-mental state”. A practical method for grading the cognitive state of patients for the clinician. J Psychiatr Res 1975;12:189-98. [CrossRef]

8. Güngen C, Ertan T, Eker E, Yașar R, Engin F. Standardize Mini Mental Test' in Türk Toplumunda Hafif Demans Tanısında Geçerlik ve Güvenilirliği. Türk Psikiyatri Derg 2002;13:273-81.

9. Küçükdeveci AA, Yavuzer G, Tennant A, Süldür N, Sonel B, Arasil T. Adaptation of the modified Barthel Index for use in physical medicine and rehabilitationin Turkey. Scand J Rehabil Med 2000;32:87-92.

10. Ware JE. SF-36 Health Survey Update. In: Maruish ME, editor. The Use of Psychological Testing for Treatment Planning and Outcomes Assessment. $3^{\text {rd }}$ ed. USA: Lawrence Erlbaum Associates 2004. p. 693 718.

11. Demirsoy C. The MOS-SF 36 health survey: A validation studt with a Turkish sample. [Master Thesis]. İstanbul: Boğaziçi University; 1999.

12. Ohene-Frempong K, Weiner SJ, Sleeper LA, Miller ST, Embury S, Moohr JW, et al. Cerebrovascular accidents in sickle cell disease: rates and risk factors. Blood 1998;91:288-94.

13. Wade DT. Personal physical disability. In: Wade DT, editör. Measurement in Neurological Rehabilitation. Oxford: Oxford University Pres; 1992. p. 71-82.

14. Collen FM, Wade DT, Robb GF, Bradshaw CM. The Rivermead Mobility Index: a further development of the Rivermead Motor Assessment. Int Disabil Stud 1991;13:50-4. [CrossRef]

15. Berg KO, Wood-Dauphinee SL, Williams JI, Maki B. Measuring balance in the elderly: validation of an instrument. Can J Public Health 1992;83 Suppl 2:S7-11.

16. Berg K, Wood-Dauphinee S, Williams JI. The Balance Scale: reliability assessment with elderly residents and patients with an acute stroke. Scand J Rehabil Med 1995;27:27-36.

17. Şahin F, Büyükavcı R, Sağ S, Doğu B, Kuran B. Reliability and Validity of the Turkish Version of the Berg Balance Scale in Patients With Stroke. Turk Phys Med and Rehab 2013;59:170-5.

18. Beaton DE, Bombardier C, Guillemin F, Ferraz MB. Guidelines for the process of cross-cultural adaptation of self-report measures. Spine (Phila Pa 1976) 2000;25:3186-91. [CrossRef]

19. Guillemin F. Cross-cultural adaptation and validation of health status measures. Scand J Rheumatol 1995;24:61-3. [CrossRef]

20. Petersen MA, Groenvold M, Bjorner JB, Aaronson N, Conroy T, Cull 
A, et al; European Organisation for Research and Treatment of Cancer Quality of Life Group. Use of differential item functioning analysis to assess the equivalence of translations of a questionnaire. Qual Life Res 2003;12:373-85. [CrossRef]

21. Küçükdeveci AA. Osteoartritte işlevsel değerlendirme ölçütleri. Turk J Geriatrics 2011;14:37-44.

22. Aboderin I, Venables G. Stroke management in Europe. Pan European Consensus Meeting on StrokeManagement. J Intern Med 1996;240:173-80. [CrossRef]

23. Bohannon RW. Lateral trunk flexion strength: impairment, measurement reliability and implications following unilateral brain lesion. Int J Rehabil Res 1992;15:249-51. [CrossRef]

24. Brinkman J, Kuypers HG. Splitbrain monkeys: cerebral control of ipsilateral and contralateral arm, hand, and finger movements. Science 1972;176:536-9. [CrossRef]

25. Taoka M, Toda T, Iwamura Y. Representation of the midline trunk, bilateral arms, and shoulders in the monkey postcentral somatosensory cortex. Exp Brain Res 1998;123:315-22. [CrossRef]

26. Côté R, Hachinski VC, Shurvell BL, Norris JW, Wolfson C. The Canadian Neurological Scale: a preliminary study in acute stroke. Stroke 1986;17:731-7. [CrossRef]
27. Kottke FJ. Neurophysiologic therapy for stroke. In: Licht S, editor. Stroke and Its Rehabilitation. Baltimore: Waverly Press; 1975 p. 256324.

28. Verheyden G, Nieuwboer A, Feys H, Thijs V, Vaes K, De Weerdt W. Discriminant ability of the Trunk Impairment Scale: A comparison between stroke patients and healthy individuals. Disabil Rehabil 2005;27:1023-8. [CrossRef]

29. Verheyden G, Vereeck L, Truijen S, Troch M, Lafosse C, Saeys W, et al. Additional exercises improve trunk performance after stroke: a pilot randomized controlled trial. Neurorehabil Neural Repair 2009;23:281-6. [CrossRef]

30. Verheyden G, Nieuwboer A, Mertin J, Preger R, Kiekens C, De Weerdt W. The Trunk Impairment Scale: a new tool to measure motor impairment of the trunk after stroke. Clin Rehabil 2004;18:326-34. [CrossRef]

31. Verheyden G, Hughes J, Jelsma J, Nieuwboer A, De Weerdt W. Assesing motor impairment of the trunk in patients with traumatic brain injury: reliability and validity of the trunk impairment scale. S Afr J Physiother 2006;62:23-8. [CrossRef]

32. Verheyden G, Nieuwboer A, De Wit L, Feys H, Schuback B, Baert I, et al. Trunk performance after stroke: an eye catching predictor of functional outcome. J Neurol Neurosurg Psychiatry 2007;78:694-8. [CrossRef] 